\title{
SOLUTIONS FOR SINGULAR ELLIPTIC SYSTEMS INVOLVING HARDY-SOBOLEV CRITICAL NONLINEARITY
}

\author{
LING DING AND SHI-WU XIAO
}

Abstract. In this paper, we deal with a class of singular elliptic system with Hardy-Sobolev critical nonlinearity. The existence and multiplicity of solutions for this system are obtained by the variational methods and some analysis techniques.

Mathematics subject classification (2010): 35J60, 35J20, 35B33.

Keywords and phrases: elliptic systems, Hardy terms, critical Hardy-Sobolev exponents.

\section{REFERENCES}

[1] C. O. Alves and D. G. De Figueiredo, Nonvariational elliptic systems, Discr. Contin. Dyn. Syst., 8 (2002), 289-302.

[2] C. O. Alves, D. C. De Morais Filho And M. A. S. Souto, On systems of elliptic equations involving subcritical or critical Sobolev exponents, Nonlinear Anal., 42 (2000), 771-787.

[3] H. Brezis AND L. Nirenberg, Positive solutions of nonlinear elliptic equations involving critical Sobolev exponents, Comm. Pure Appl. Math., 36 (1983), 437-477.

[4] N. Ghoussoub AND C. YUAN, Multiple solutions for quasi-linear PDEs involving the critical Sobolev and Hardy exponents, Trans. Amer. Math. Soc., 352 (2000), 5703-5743.

[5] P. G. HAN, High-energy positive solutions for a critical growth Dirichlet problem in noncontractible domains, Nonlinear Anal., 60 (2005), 369-387.

[6] P. G. HAN, The effect of the domain topology on the number of positive solutions of an elliptic system involving critical Sobolev exponents, Houston J. Math., 32 (2006), 1241-1257.

[7] P. G. HAN, Strongly indefinite systems with critical Sobolev exponents and weights, Appl. Math. Lett., 17 (2004), 909-917.

[8] D. S. Kang And S. J. Peng, Positive solutions for singular critical elliptic problems, Appl. Math. Lett., 17 (2004), 411-416.

[9] Z. X. LIU AND P. G. HAN, Existence of solutions for singular elliptic systems with critical exponents, Nonlinear Anal., 69 (2008), 2968-2983.

[10] Y. LoU, Necessary and sufficient condition for the existence of positive solutions of certain cooperative system, Nonlinear Anal., 26 (1996), 1079-1095.

[11] P. H. Rabinowitz, Minimax methods in critical point theory with applictions to differential equations, CBMS Reg. Conf. Series . Math., 65, Amer. Math. Soc., Providence, RI, 1986.

[12] P. H. ZHAO AND X. Y. WANG, The existence of positive solutions of elliptic system by a linking theorem on product space, Nonlinear Anal., 56 (2004), 227-240. 